\title{
One year in circulation - an exciting journey for ADMET \& DMPK
}

\author{
Kin Tam
}

Editor: $A D M E T \& D M P K$

E-mail: kin tam@iapchem.org

It is now almost a year since ADMET \& DMPK launched. The current issue will be the final one for 2013. We are happy to report that it has been a very exciting year for the journal. In particular, we have published a range of articles, such as book reviews, original scientific papers, perspectives, opinions and mini reviews covering different areas of interest to the journal. This reflects the diversity of the type of articles that enables the exchange of knowledge and shared learning. We would like to take this opportunity to offer thanks for all the help and hard work from our editorial team for supporting the journal.

\section{Meet the ADMET \& DMPK editorial team}

Mandić has written an excellent review on a book entitled "Absorption and Drug Development. Solubility, Permeability and Charge State. 2nd Edition" by the leading expert in the area, Alex Avdeef [1]. He has given a good overview of the book, and remarked that "this book is essential literature on the desk of the pharmaceutical scientist". Next, Gabelica Marković has published a book review on "Physico Chemical Methods in Drug Discovery and Development", edited by Zoran Mandić [2]. This book was indeed a collection from some selected high quality presentations summarizing the key methods and techniques in pharmaceutical research from the $2^{\text {nd }}$ World Conference on Physico Chemical Methods in Drug Discovery and Development held in Zadar in 2011. With regard to the original scientific article, Takács-Novák et al. have published their study of the acid-based properties of Lisinopril, a tetraprotic ampholyte ACE inhibitor [3]. NMR-pH titration was used to assign the constants to the functional groups and for the examination of site-specific, submolecular basicities of the molecule. The microconstants and macroconstants were reported and used to help the interpretation of the pharmacokinetic and pharmacodynamic properties of lisinopril. Moreover, Avdeef et al has described a versatile computer program, $p D I S O L-X^{T M}$, to process salt solubility data [4]. This novel approach does not require any explicitly derived equations, and is able to deal with other equilibrium process, such as aggregate formation, as well as performs adjustment calculation in high ionic strength environment. We envisage that $p D I S O L-X^{T M}$ will be a powerful tool for our preformulation colleagues during salt selection. Hong has written a perspective article to discuss the ADME tests that should be conducted in preclinical studies, with a particular emphasis on the data needed to support IND filing [5]. In the article, the author has shared his views and opinion based on his many years of experience in drug discovery, CROs and IND filing, which is a must-read for readers who want to learn 
more about pharmaceutical development. Tam has published two mini review articles on the role of doxorubicin in hepatocellular carcinoma [6], and the estimation of first-in-man doses with particular emphasis on oncology drugs [7]. Finally, $W u$ et al. have published an opinion article concerning the regulatory control of medical device containing nanomaterial [8]. This is an emerging area where the product safety due to the presence of nanomaterial could be a concern, especially for manufacturing staff, customers and end users. The authors have pointed out that regulatory regulations are important for this kind of medical device.

Despite the promising development we have seen this year, many challenges remain. For instance, it is generally difficult for new journals to attract a good number of high quality paper submissions in the first few years. No doubt ADMET \& DMPK is suffering from the same problem. Hopefully this will become less of an issue next year, as some of our academic/industrial colleagues have already expressed interests and/or committed to submit their articles to our journal.

It is believed that $A D M E T \& D M P K$ has a unique position in the open literature [9]. To aid the rapid scientific discussion, the journal will seek to publish commentary/correspondence articles where research groups could engage in the debate on a particular topic of interest. Moreover, we plan to publish a special issue highlighting the applications of electrochemical techniques in ADMET studies. It is our expectation that ADMET \& DMPK will become a platform for all scientists working in ADMET and DMPK areas to publish their research findings in a timely manner.

Acknowledgements: We thank IAPC Publishing for supporting the running cost of the journal and hosting it on their website.

\section{References}

[1] Z. Mandić, AMDET \& DMPK 1 (2013) 3-5.

[2] V.Gabelica Marković, AMDET \& DMPK 1 (2013) 17-18.

[3] K. Takács-Novák, K. Deák, S. Béni, G. Völgyi, AMDET \& DMPK 1 (2013) 6-16.

[4] G. Völgyi, A. Marosi, K. Takács-Novák, A. Avdeef, AMDET \& DMPK 1 (2013) 48-62.

[5] H. Wan, AMDET \& DMPK 1 (2013) 19-28.

[6] K. Tam, AMDET \& DMPK 1 (2013) 29-44.

[7] K. Tam, AMDET \& DMPK 1 (2013) 63-75.

[8] E. Wu, C. Chan, A.T.W. Li, AMDET \& DMPK 1 (2013) 76-81.

[9] K. Tam, AMDET \& DMPK 1 (2013) 1-2.

C2013 by the authors; licensee IAPC, Zagreb, Croatia. This article is an open-access article distributed under the terms and conditions of the Creative Commons Attribution license (http://creativecommons.org/licenses/by/3.0/) (cc)) EY 